\title{
Some identities involving multiplicative semiderivations on ideals
}

\author{
Öznur Gölbaşı*(D), Zeliha Bedir (D) \\ Cumhuriyet University, Faculty of Science, Department of Mathematics, Sivas, Turkey
}

\begin{abstract}
Let $R$ be a prime ring and $I$ be a nonzero ideal of $R$. A mapping $d: R \rightarrow R$ is called a multiplicative semiderivation if there exists a function $g: R \rightarrow R$ such that (i) $d(x y)=$ $d(x) g(y)+x d(y)=d(x) y+g(x) d(y)$ and (ii) $d(g(x))=g(d(x))$ hold for all $x, y \in R$. In the present paper, we shall prove that $[x, d(x)]=0$, for all $x \in I$ if any of the followings holds: i) $d(x y) \pm x y \in Z$, ii) $d(x y) \pm y x \in Z$, iii) $d(x) d(y) \pm x y \in Z$, iv $) d(x y) \pm d(x) d(y) \in Z$, viii) $d(x y) \pm d(y) d(x) \in Z$, for all $x, y \in I$. Also, we show that $R$ must be commutative if $d(I) \subseteq Z$.
\end{abstract}

Mathematics Subject Classification (2020). 16W25, $16 \mathrm{U} 80$

Keywords. prime rings, semiderivation, multiplicative semiderivation

\section{Introduction}

Throughout this paper, $Z$ will denote the center of an associative ring $R$. For $x, y \in R$, a ring $R$ is said to be prime if whenever $x R y=(0)$ implies $x=0$ or $y=0$. The concept of derivation was first time introduced by Posner in [11]. An additive mapping $d: R \rightarrow R$ is called a derivation if $d(x y)=d(x) y+x d(y)$ holds for all $x, y \in R$. During the past few years, several authors have been studying the commutativity of prime rings.

The present paper has been motivated by the works of Ashraf and Rehman [2] and Dhara and Ali [8]. Ashraf and Rehman proved that for a prime ring $R$ with a nonzero ideal $U$ of $R$ if $d$ is a derivation of $R$ such that $d(x y) \pm x y \in Z$, for all $x, y \in U$, then $R$ is commutative. Also, Bell and Kappe showed in [3] that a derivation $d$ of a prime ring $R$ acting as a homomorphism or an anti-homomorphism on a nonzero right ideal of $R$ must vanish, i.e., $d=0$ on $R$.

In [4], Bergen introduced the following notion: An additive mapping $d: R \rightarrow R$ is called a semiderivation if there exists a function $g: R \rightarrow R$ such that (i) $d(x y)=d(x) g(y)+$ $x d(y)=d(x) y+g(x) d(y)$ and (ii) $d(g(x))=g(d(x))$ hold for all $x, y \in R$. In case $g$ is an identity map of $R$, then all semiderivations associated with $g$ are merely derivations. Other main motivating examples are of the form $d=g-1$ where $g$ is any endomorphism of $R$ such that $g \neq 1$. Then $d$ is a semiderivation associated with the map $g$ which is not a derivation. In case $R$ is prime and $d \neq 0$, it has been shown by Chang [6] that $g$ must necessarily be a ring endomorphism.

\footnotetext{
*Corresponding Author.

Email addresses: ogolbasi@cumhuriyet.edu.tr (Ö. Gölbaşı), zelihabedir@cumhuriyet.edu.tr (Z. Bedir)

Received: 25.11.2019; Accepted: 04.02.2021
} 
A multiplicative derivation of $R$ is a mapping $d: R \rightarrow R$ which satisfies $d(x y)=$ $d(x) y+x d(y)$ for all $x, y \in R$. So a multiplicative derivation will be a derivation when it is an additive map. Recently, some well-known results concerning prime and semiprime rings have been proved for multiplicative derivations. Hence, one may observe that the concept of multiplicative semiderivations includes the concept of derivations and the left multipliers (i.e., $d(x y)=d(x) y$ for all $x, y \in R$ ). For details, we refer to [7], a work of Daif, which was motivated by the work of Martindale [10]. Further, Goldman and Semrl gave the complete description of these maps in [9]. Obviously, for $g=1$ a semiderivation is an ordinary derivation and every derivation is a multiplicative derivation. But the converse is not true in general. So, it should be interesting to extend some results concerning these notions to multiplicative semiderivations.

Our aim in this work is to investigate the commutativity of prime rings admitting multiplicative semiderivations on nonzero ideal of $R$. We extend the above mentioned results of Ashraf and Rehman [2] and Dhara and Ali [8] for multiplicative semiderivations on nonzero ideal of $R$.

\section{Results}

Throughout the paper, we assume that $R$ is a prime ring, $I$ is a nonzero ideal of $R$ and $d$ is a multiplicative semiderivation of $R$ associated with a nonzero surjective map $g$ of $R$.

Also, for $x, y \in R$ the symbol $[x, y]$ stands for Lie commutator which is defined as $x y-y x$ and it satisfies the basic commutator identities:

$$
\begin{aligned}
& {[x, y z]=y[x, z]+[x, y] z} \\
& {[x y, z]=[x, z] y+x[y, z] .}
\end{aligned}
$$

Lemma 2.1 ([5, Lemma 4.2]). Let $R$ be a prime ring, $I$ be a nonzero ideal of $R$ and $d$ be a nonzero derivation of $R$. If $d(I) \subseteq Z$, then $R$ is commutative.

Lemma 2.2 ([1, Theorem 2.4]). Let $R$ be a semiprime ring, $I$ be a nonzero ideal of $R$ and $d$ be a multiplicative semiderivation associated with a nonzero epimorphism $g$ of $R$. If $d(x y) \pm x y=0$, for all $x, y \in I$, then $[x, d(x)]=0$, for all $x \in I$.

Lemma 2.3 ([1, Theorem 5]). Let $R$ be a semiprime ring, $I$ be a nonzero ideal of $R$ and $d$ be a multiplicative semiderivation associated with a nonzero epimorphism $g$ of $R$. If $d(x) d(y) \pm x y=0$, for all $x, y \in I$, then $[x, d(x)]=0$, for all $x \in I$.

Lemma 2.4 ([1, Theorem 7]). Let $R$ be a semiprime ring, $I$ be a nonzero ideal of $R$ and $d$ be a multiplicative semiderivation associated with a nonzero epimorphism $g$ of $R$. If $d(x y)= \pm d(x) d(y)$, for all $x, y \in I$, then $[x, d(x)]=0$, for all $x \in I$.

Lemma 2.5 ([1, Theorem 8]). Let $R$ be a semiprime ring, $I$ be a nonzero ideal of $R$ and $d$ be a multiplicative semiderivation associated with a nonzero epimorphism $g$ of $R$. If $d(x y)= \pm d(y) d(x)$, for all $x, y \in I$, then $[x, d(x)]=0$, for all $x \in I$.

Lemma 2.6. Let $R$ be a prime ring, $I$ be a nonzero ideal of $R$ and $d$ be a multiplicative semiderivation associated with a nonzero map $g$ of $R$ and $a \in R$. If ad $(I)=(0)$, then $a=0$ or $d=0$.

Proof. By the hypothesis, we have $a d(x r)=0$, for all $x \in I, r \in R$, and so

$$
a d(x) g(r)+\operatorname{axd}(r)=0 .
$$

Using the hypothesis, we get

$$
a I d(r)=(0), \text { for all } r \in R .
$$

By the primeness of $R$, we find that

$$
a=0 \text { or } d=0 .
$$


Lemma 2.7. Let $R$ be a prime ring. If $d$ is a nonzero multiplicative semiderivation associated with a map $g$ of $R$, then

$$
g(x y)=g(x) g(y), \text { for all } x, y \in R .
$$

Proof. For all $x, y, z \in R$, by the definition of $d$, we have

$$
\begin{aligned}
d(x(y z)) & =d(x) y z+g(x) d(y z) \\
& =d(x) y z+g(x) d(y) z+g(x) g(y) d(z) .
\end{aligned}
$$

On the other hand, we get

$$
\begin{aligned}
d((x y) z) & =d(x y) z+g(x y) d(z) \\
& =d(x) y z+g(x) d(y) z+g(x y) d(z) .
\end{aligned}
$$

Comparing the last two equations, we obtain that

$$
g(x y) d(z)=g(x) g(y) d(z), \text { for all } x, y, z \in R .
$$

That is

$$
(g(x y)-g(x) g(y)) d(z)=0, \text { for all } x, y, z \in R .
$$

By Lemma 2.6, we get $g(x y)=g(x) g(y)$, for all $x, y \in R$. The proof is completed.

Theorem 2.8. Let $R$ be a prime ring, $I$ be a nonzero ideal of $R$ and $d$ be a nonzero multiplicative semiderivation associated with a map $g$ of $R$. If $d(I) \subseteq Z$, then $R$ is a commutative ring.

Proof. By the hypothesis, we have $d(r x) \in Z$, for $x \in I, r \in R$. Hence we get

$$
\begin{aligned}
d(r x) s & =s d(r x) \\
d(r) x s+g(r) d(x) s & =s d(r) x+s g(r) d(x)
\end{aligned}
$$

and so

$$
d(r) x s-s d(r) x=d(x)[s, g(r)], \text { for all } x \in I, r, s \in R .
$$

Replacing $g(r)$ by $s$ in (2.1), we get

$$
d(r) x g(r)=g(r) d(r) x, \text { for all } x \in I, r \in R .
$$

Writing $x s$ for $x$ in (2.2) and using (2.2), we obtain that

$$
d(r) x[s, g(r)]=0, \text { for all } x \in I, r, s \in R .
$$

Then, the primeness of $R$ yields

$$
d=0 \text { or } g(r) \in Z, \text { for all } r \in R .
$$

Let us take $g(r) \in Z$, for all $r \in R$. By the hypothesis, we get

$$
d(x y)=d(x) y+g(x) d(y) \in Z
$$

and so

$$
d(x) y \in Z, \text { for all } x, y \in I .
$$

By the primeness of $R$ and $d \neq 0$, we find that $R$ is a commutative ring. This completes the proof.

Theorem 2.9. Let $R$ be a prime ring, $I$ be a nonzero ideal of $R$ and $d$ be a nonzero multiplicative semiderivation associated with a nonzero surjective map $g$ of $R$. If $d(x y) \pm$ $x y \in Z$, for all $x, y \in I$, then $[x, d(x)]=0$, for all $x \in I$. 
Proof. By our hypothesis, we get

$$
d(x y) \pm x y \in Z, \text { for all } x, y \in I .
$$

Writing $y r, r \in R$ for $y$ in this equation, we have

$$
d(x y r) \pm x y r=d(x y) r+g(x y) d(r) \pm x y r,
$$

which implies

$$
(d(x y) \pm x y) r+g(x y) d(r) \in Z, \text { for all } x, y \in I, r \in R .
$$

Commuting both sides with $r \in R$, we have

$$
[(d(x y) \pm x y) r+g(x y) d(r), r]=0 .
$$

So the hypothesis yields that

$$
[g(x y) d(r), r]=0, \text { for all } x, y \in I, r \in R .
$$

Replacing $s x, s \in R$ by $x$ in (2.4) and using this equation, we get

$$
[g(s), r] g(x y) d(r)=0
$$

which gives

$$
[t, r] g(x y) d(r)=0, \text { for all } x, y \in I, r, t \in R,
$$

by the surjectivity of $g$. Taking $t s, s \in R$ instead of $t$ in this equation, we find that

$$
[t, r] s g(x y) d(r)=0 \text {, }
$$

and so

$$
[t, r] R g(x y) d(r)=0, \text { for all } x, y \in I, r, t \in R .
$$

Since $R$ is a prime ring, we get

$$
R \text { is commutative or } g(x y) d(r)=0 \text {, for all } x, y \in I, r \in R \text {. }
$$

Now we assume that $g(x y) d(r)=0$, for all $x, y \in I, r \in R$. By Lemma 2.6, we have $g(x y)=0$, for all $x, y \in I$. Using this in (2.3), we obtain that

$$
(d(x y) \pm x y) r \in Z \text {, for all } x, y \in I, r \in R .
$$

By the primeness of $R$ and $d(x y) \pm x y \in Z$, we have

$$
d(x y) \pm x y=0 \text {, for all } x, y \in I \text { or } R \text { must be commutative. }
$$

If $R$ is commutative, then we have $[x, d(x)]=0$, for all $x \in I$. If $d(x y) \pm x y=0$, for all $x, y \in I$, then we get $[x, d(x)]=0$, for all $x \in I$ by Lemma 2.2. This completes the proof.

Theorem 2.10. Let $R$ be a prime ring, $I$ be a nonzero ideal of $R$ and $d$ be a nonzero multiplicative semiderivation associated with a nonzero surjective map $g$ of $R$. If $d(x y) \pm$ $y x \in Z$, for all $x, y \in I$, then $[x, d(x)]=0$, for all $x \in I$.

Proof. By our hypothesis, we get

$$
d(x(y z)) \pm(y z) x \in Z, \text { for all } x, y, z \in I
$$

and

$$
d((x y) z) \pm z(x y) \in Z, \text { for all } x, y, z \in I .
$$

Subtracting these two equations yields that

$$
[y, z x] \in Z \text {, for all } x, y, z \in I .
$$

Hence we get $\delta_{z x}(I) \subseteq Z$ where $\delta_{z x}(y)=[y, z x]$ is an inner derivation. By Lemma 2.1, we obtain that $R$ is commutative or $\delta_{z x}=0$. If $\delta_{z x}=0$, then we have $[y, z x]=0$, for all $x, y, z \in I$. Writing $r z, r \in R$ for $z$ in this equation and using this, we obtain that

$$
[y, r] z x=0, \text { for all } x, y, z \in I, r \in R .
$$


By the primeness of $R$, we find that $R$ is commutative and so $[x, d(x)]=0$, for all $x \in I$. This completes the proof.

Theorem 2.11. Let $R$ be a prime ring, $I$ be a nonzero ideal of $R$ and $d$ be a nonzero multiplicative semiderivation associated with a nonzero surjective map $g$ of $R$. If $d(x) d(y) \pm$ $x y \in Z$, for all $x, y \in I$, then $[x, d(x)]=0$, for all $x \in I$.

Proof. Taking $y z$ instead of $y$ in the hypothesis, we have

$$
d(x) d(y z) \pm x y z \in Z
$$

which implies

$$
(d(x) d(y) \pm x y) z+d(x) g(y) d(z) \in Z, \text { for all } x, y, z \in I .
$$

Taking commutation of this term with $z$ and using our hypothesis, we have

$$
[d(x) g(y) d(z), z]=0, \text { for all } x, y, z \in I .
$$

Replacing $z y$ by $y$ in (2.8), we get

$$
[d(x) g(z) g(y) d(z), z]=0 \text {, for all } x, y, z \in I .
$$

Writing $x z$ for $x$ in (2.8) and using (2.9), we obtain that

$$
[x d(z) g(y) d(z), z]=0 .
$$

By (2.8), we have

$$
[x, z] d(z) g(y) d(z)=0, \text { for all } x, y, z \in I .
$$

Putting $r y, r \in R$ instead of $y$ in this equation, we get

$$
[x, z] d(z) g(r) g(y) d(z)=0, \text { for all } x, y, z \in I, r \in R .
$$

Using the surjectivity of $g$, the last equation can be written as

$$
[x, z] d(z) R g(y) d(z)=(0) \text {, for all } x, y, z \in I,
$$

and so

$$
[x, z] d(z)=0 \text { or } g(y) d(z)=0 \text {, for all } x, y, z \in I .
$$

Now, we assume that $[x, z] d(z)=0$, for all $x, z \in I$. Writing $x y$ instead of $x$ in this equation, we have $[x, z] y d(z)=0$, for all $x, y, z \in I$, and so, $z \in Z$ or $d(z)=0$. Hence we arrive at $d(z) \in Z, z \in I$ for any cases.

On the other hand, let $g(y) d(z)=0$, for all $y, z \in I$. Replacing $y r, r \in R$ by $y$ in this equation, we get $g(y) g(r) d(z)=0$, for all $y, z \in I, r \in R$, and so $g(y) R d(z)=(0)$, for all $y, z \in I$. Since $R$ is a prime ring, we have $d(z)=0$ or $g(I)=(0)$. Hence we get $d(z) \in Z$, for all $z \in I$ or $g(I)=(0)$.

If $d(z) \in Z$, for all $z \in I$, then $R$ must be commutative by Theorem 2.8 and it implies that $[x, d(x)]=0$, for all $x \in I$.

Now, we assume that $g(I)=(0)$. Considering (2.7), we obtain that

$$
(d(x) d(y) \pm x y) z \in Z \text {, for all } x, y, z \in I .
$$

Since $d(x y) \pm x y \in Z$, we have

$$
d(x) d(y) \pm x y=0 \text {, for all } x, y \in I \text { or } R \text { must be commutative. }
$$

If $d(x) d(y) \pm x y=0$, for all $x, y \in I$, then we get $[x, d(x)]=0$, for all $x \in I$ by Lemma 2.3. Hence we get the required result.

Theorem 2.12. Let $R$ be a prime ring, $I$ be a nonzero ideal of $R$ and $d$ be a nonzero multiplicative semiderivation associated with a nonzero surjective map $g$ of $R$. If $d(x y) \pm$ $d(x) d(y) \in Z$, for all $x, y \in I$, then $[x, d(x)]=0$, for all $x \in I$. 
Proof. By our hypothesis, we get

$$
d(x y) \pm d(x) d(y) \in Z, \text { for all } x, y \in I .
$$

Replacing $y z$ by $y$ in this equation, we have

$$
d(x y) z+g(x y) d(z) \pm d(x) d(y) z \pm d(x) g(y) d(z) \in Z
$$

and so

$$
(d(x y) \pm d(x) d(y)) z+g(x y) d(z) \pm d(x) g(y) d(z) \in Z, \text { for all } x, y, z \in I .
$$

Commuting (2.11) with $z \in I$ yields that

$$
[g(x y) d(z) \pm d(x) g(y) d(z), z]=0 \text {, for all } x, y, z \in I .
$$

Writing $x z$ by $x$ in (2.12) and using (2.12), we find that

$$
[x d(z) g(y) d(z), z]=0 \text {, for all } x, y, z \in I .
$$

Replacing $r x, r \in R$ by $x$ in (2.13) and using this equation, we have

$$
[r, z] \operatorname{Id}(z) g(y) d(z)=(0) \text {, for all } y, z \in I, r \in R .
$$

By the primeness of $R$, we obtain that

$$
z \in Z \text { or } d(z) g(y) d(z)=0, \text { for all } y, z \in I \text {. }
$$

If $z \in Z$, then $d(z) \in Z$.

Let us assume that $d(z) g(y) d(z)=0$, for all $y, z \in I$. Taking $y r, r \in R$ instead of $y$ in this equation and using the fact that $g$ is surjective, we arrive at $d(z)=0$ or $d(z) g(y)=0$, and so $d(z) g(y)=0$, for all $y, z \in I$. Again writing $r y, r \in R$ for $y$ in this equation, we have

$$
d(z)=0 \text { or } g(y)=0 \text {, for all } y, z \in I .
$$

If $d(z)=0$, then we get $d(z) \in Z$, for all $z \in I$. Hence we arrive at

$$
d(z) \in Z \text { or } g(y)=0 \text {, for all } y, z \in I .
$$

Now, if $d(z) \in Z$, then $R$ is commutative by Theorem 2.8 , and so $[x, d(x)]=0$, for all $x \in I$.

On the other hand, assuming $g(y)=0$, for all $y \in I$ implies that

$$
(d(x y) \pm d(x) d(y)) z \in Z
$$

from (2.11). Since $R$ is a prime ring and $d(x y) \pm d(x) d(y) \in Z$, we have

$$
d(x y) \pm d(x) d(y)=0 \text { or } z \in Z \text {, for all } x, y, z \in I .
$$

If $z \in Z$, then $R$ is commutative, and so $[x, d(x)]=0$, for all $x \in I$. If $d(x y) \pm d(x) d(y)=0$, for all $x, y \in I$, then we get $[x, d(x)]=0$, for all $x \in I$ by Lemma 2.4. The proof is completed.

Theorem 2.13. Let $R$ be a prime ring, $I$ be a nonzero ideal of $R$ and $d$ be a nonzero multiplicative semiderivation associated with a nonzero surjective map $g$ of $R$. If $d(x y) \pm$ $d(y) d(x) \in Z$, for all $x, y \in I$, then $[x, d(x)]=0$, for all $x \in I$.

Proof. By our hypothesis, we get

$$
d(x y) \pm d(y) d(x) \in Z, \text { for all } x, y \in I .
$$

For all $x, y, z \in I$, we can write

$$
d(x(y z)) \pm d(y z) d(x) \in Z, \text { for all } x, y, z \in I
$$

and

$$
d((x y) z) \pm d(z) d(x y) \in Z, \text { for all } x, y, z \in I .
$$

Subtracting these two equations, we find that

$$
d(y z) d(x) \mp d(z) d(x y) \in Z \text {, for all } x, y, z \in I .
$$


We can write this equation as

$$
(d(y z)-d(z) d(y)) d(x)+d(z)(d(y) d(x) \mp d(x y)) \in Z, \text { for all } x, y, z \in I .
$$

Commuting this term with $d(x)$ and using the hypothesis, we find that

$$
[d(z), d(x)](d(y) d(x) \mp d(x y))=0, \text { for all } x, y, z \in I .
$$

Since $d(x y) \pm d(y) d(x) \in Z$, for all $x, y \in I$ we have

$$
[d(z), d(x)]=0 \text { or } d(y) d(x) \pm d(x y)=0 \text {, for all } x, y, z \in I .
$$

If $d(y) d(x) \pm d(x y)=0$, for all $x, y \in I$, then we get $[x, d(x)]=0$, for all $x \in I$ by Lemma 2.5 .

If $[d(z), d(x)]=0$, then $d(x) d(z)=d(z) d(x)$, for all $x, z \in I$. Using this in our hypothesis, we get $d(x y) \pm d(x) d(y) \in Z$, for all $x, y \in I$. It implies that $[x, d(x)]=0$, for all $x \in I$ by Theorem 2.12. This completes the proof.

\section{References}

[1] O. Ağırtıcı and Ö. Gölbaşı, Multiplicative semiderivations on ideals of semiprime rings, Palest. J. Math. 9 (2), 792-800, 2020.

[2] M. Ashraf and N. Rehman, On derivations and commutativity in prime rings, EastWest J. Math. 3 (1), 87-91, 2001.

[3] H.E. Bell and L.C. Kappe, Rings in which derivations satisfy certain algebraic conditions, Acta Math. Hungar. 53, 339-346, 1989.

[4] J. Bergen, Derivations in prime rings, Canad. Math. Bull. 26, 267-270, 1983.

[5] M. Bresar, Centralizing mappings and derivations in prime rings, J. Algebra 156, 385-394, 1983.

[6] J.C. Chang, On semiderivations of prime rings, Chinese J. Math. 12, 255-262, 1984.

[7] M.N. Daif, When is a multiplicative derivation additive, Int. J. Math. Math. Sci. 14 (3), 615-618, 1991.

[8] B. Dhara and S. Ali, On multiplicative (generalized) derivation in prime and semiprime rings, Aequationes Math. 86, 65-79, 2013.

[9] H. Goldman and P. Semrl, Multiplicative derivations on $C(X)$, Monatsh Math. 121 (3), 189-197, 1969.

[10] W.S. Martindale III, When are multiplicative maps additive, Proc. Amer. Math. Soc. 21, 695-698, 1969.

[11] E.C. Posner, Derivations in prime rings, Proc. Amer. Math. Soc. 8, 1093-1100, 1957. 